\title{
Prospects for Higgs Boson Measurements and Beyond Standard Model Physics at the High-Luminosity LHC with CMS
}

\author{
Rosamaria Venditti ${ }^{1, *}$, for the CMS Collaboration \\ ${ }^{1}$ INFN, Sezione di Bari, Via Orabona 4, Bari
}

\begin{abstract}
The High-Luminosity Large Hadron Collider (HL-LHC) is a major upgrade of the LHC, expected to deliver an integrated luminosity of up to $3000 / \mathrm{fb}$ over one decade. The very high instantaneous luminosity will lead to about 200 proton-proton collisions per bunch crossing (pileup) superimposed to each event of interest, therefore providing extremely challenging experimental conditions. The scientific goals of the HL-LHC physics program include precise measurement of the properties of the recently discovered standard model Higgs boson and searches for beyond the standard model physics (heavy vector bosons, SUSY, dark matter and exotic long-lived signatures, to name a few). In this contribution we will present the strategy of the CMS experiment to investigate the feasibility of such search and quantify the increase of sensitivity in the HL-LHC scenario.
\end{abstract}

\section{Introduction}

This paper presents physics studies and motivations that lead the development of the strategy of the future operation of the LHC collider with high luminosity (HL-LHC), also called LHC Phase-2. After the third long shutdown of the machine, that will take place around 2023, the instantaneous luminosity will be increased up to five times the design value, with 40 $\mathrm{MHz}$ operation (one bunch crossing every $25 \mathrm{~ns}$ ) at a center of mass energy of $14 \mathrm{TeV}$. This yields challenging conditions for the CMS detector: up to 200 overlaying (pileup) events, high rates and high radiation levels, especially in the forward regions. An upgrade of the detectors is mandatory, in order to fully exploit the augmented luminosity and efficiently cope the new data taking conditions. It is foreseen that the total accumulated luminosity will reach $3000 / \mathrm{fb}$ of data, corresponding to ten times the data expected at the end of Phase1. This environment offers great opportunities to shade some light on some unexplored or not fully explored physics searches. Test of the Standard Model can be performed with sufficient precision in the Higgs sector, by accurately measuring the couplings to fermions and bosons. Searches for the associated production of two Higgs boson and measurement of the trilinear Higgs boson coupling can be a unique opportunity to test the Higgs potential and is sensitive to the presence of new physics that can be explored in both resonant and non-resonant searches. The measurement of the differential cross-section of the Higgs boson is also an interesting test-bench of the Standard Model, providing that a percent precision can be reached.

*e-mail: rosamaria.venditti@cern.ch 
The SM does not provide answers to the remaining questions. Those require new physics. In fact, the scalar nature of the particle, presents theoretical challenges. Radiative corrections to the Higgs should cause the mass to increase to very high values. New physics must appear at masses not too far from $1 \mathrm{TeV}$ to cancel this growth. Deviations from perfect SM behavior because of its interaction with other forms of matter, including dark matter, could answer some very fundamental questions, such as the origin of the matter-antimatter asymmetry of the universe. The Supersymmetric (SUSY) particles, foreseen by the homonymous theory and still unobserved by date up to the TeV scale, could offer the advantage to cancel the the growth of the Higgs mass from radiative corrections. The rate of production and the characteristics of decays of SUSY particles depend on their mass spectrum, which is not predicted, so the search has to investigate many possibilities. SUSY also predicts several more Higgstype particles. Searches for these have also been undertaken but so far no additional Higgs bosons have been found. In what follows, the study of some selected benchmark physics processes, both in the Higgs sector and in the search for New Physics at the HL-LHC will be presented.

As mentioned above, a general upgrade of the detectors is crucial to maximize physics potential and maintain a good particle reconstruction in the harsh environment of HL-LHC. The planned upgrades of the CMS experiment in view of the HL-LHC phase will take place around 2023 and are fully described in [1], [2], [3], [4].

\section{Higgs Boson Signal Strength}

The present status of the Higgs strength and couplings precision measurement performed by the CMS experiment is summarized in [5], where the the main decay channels of the Higgs bosons are exploited (WW, ZZ, $\gamma \gamma, \mu \mu, \tau \tau, \bar{b} b$ ). Using the $2016 \mathrm{CMS}$ dataset (36/fb) 10$20 \%$ uncertainties are reached on the main parameters, and the signal strength relative to the standard model is close to unity [5].

The HL-LHC will be a Higgs factory: over 170 million Higgs bosons will be produced during the whole data taking period in $3000 / \mathrm{fb}$. The increased size of this dataset will allow to achieve high precision measurements, down to the level of a few percents. The results summarized in this report are based on CMS public measurements performed using the 2015 and early 2016 proton-proton datasets, projected to larger datasets of 3000/fb assuming a center of mass energy of $13 \mathrm{TeV}$, thus using a smaller dataset with respect to the one used for extracting the measurement in [5]. The projections on the Higgs coupling are presented under different scenarios assumed for the size of systematic uncertainties, which are expected to bracket a realistic extrapolation. The incorporation of the performance of the upgraded detectors and the effect of higher pileup conditions are included in both scenarios:

- S1+ : All systematic uncertainties are kept constant with integrated luminosity.

- $\mathrm{S} 2+$ : Theoretical uncertainties scaled down by a factor $1 / 2$, while experimental systematic uncertainties are scaled down by the square root of the integrated luminosity until they reach a defined lower limit based on estimates of the achievable accuracy with the upgraded detector.

The rates of Higgs boson production and decay into $Z Z \rightarrow 4 l$ and $\gamma \gamma$ final states, parametrized using strength parameters $\mu$ (defined as the ratios between the observed rates and the expected ones in the SM) are showed in Fig. 1. The extrapolations show that all the couplings precisions are statistically limited, so theoretical and and experimental systematics will play a central role in the future measurements. Further improvements are expected by incorporating features of recent Run 2 analyses (e.g. $\mathrm{ttH}$ ) and the full Run 2 dataset. The benefit given by the increased dataset is clearly shown in the Higgs to top quarks coupling (accessible via 

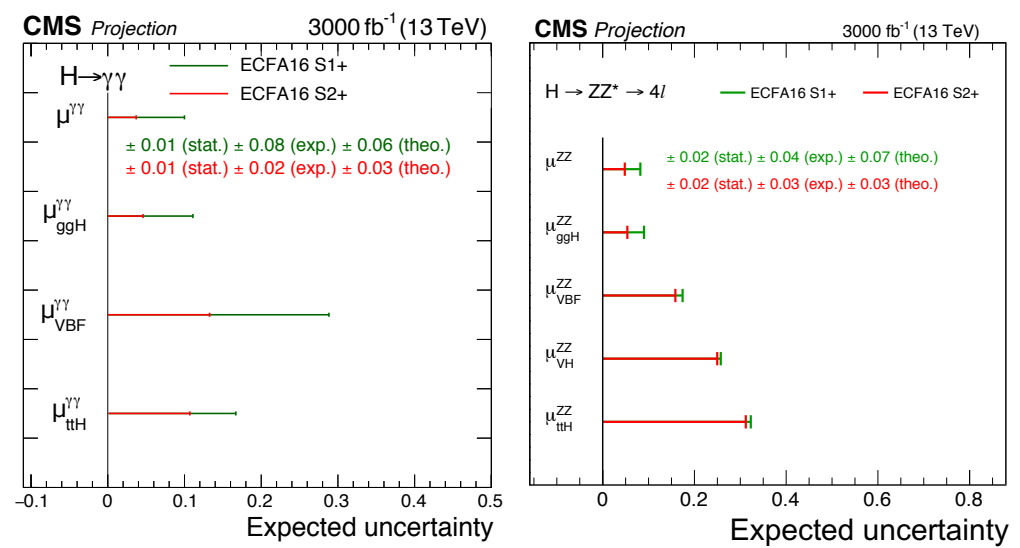

Figure 1. The projected 68\% CL uncertainties in the Higgs boson signal strength for different production modes at 3000/fb, in the $\gamma \gamma$ (top plot) and $Z Z \rightarrow 4 l$ (bottom plot) channels with $\mathrm{S} 1(+)$ in green and $\mathrm{S} 2(+)$ in red

gluon fusion production mechanism) although systematics also important. One of the main goals of HL-LHC physics program is to bring uncertainty on the rate down to $5 \%$ level

\section{Higgs differential cross-sections}

Differential cross sections provide an interesting portal to a number of physical observables. The shape of the differential cross section distribution can be tested with respect to SM expectation: it has been shown in [6] that small variation of the Higgs boson couplings with fermions, introduced by BSM processes, can lead to significant shape distortions. The differential cross section as a function of the Higgs transverse momentum is sensitive to the modifications introduced by the of effective Higgs Yukawa couplings at low $p_{T}$ and to finite top mass effects at high $p_{T}$. The present status of this measurement performed by CMS quotes a $30 \%$ to $40 \%$ uncertainties depending on the considered $p_{T}$ range [7].

The projection for the HL-LHC phase of the differential cross section measurement as a function of the transverse momentum of the Higgs boson is shown in Fig. 2. The theoretical

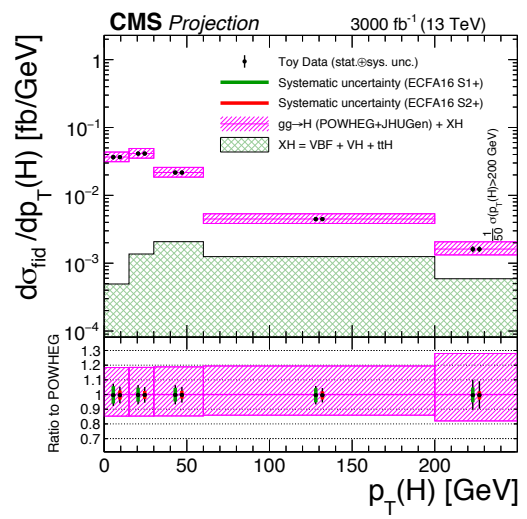

Figure 2. Projections for the differential fiducial cross section measurement of the Higgs boson transverse momentum at $3000 / \mathrm{fb}$. The theoretical uncertainty in the differential gluon fusion cross section, which does not affect the measurement, is taken at NLO and shown in magenta. The last bin represents the integrated cross section for $p_{T} \geq 200 \mathrm{GeV}$ and is scaled by 50 for presentation.

uncertainties in the total signal cross section are not relevant and the cross section is measured in a fiducial phase space closely matching the experimental acceptance. The statistical 
uncertainty of the measurements ranges from 4 to $9 \%$ depending on the $p_{T}$ bin. The las bin $\left(p_{T} \geq 200 \mathrm{GeV}\right)$ is still dominated by the statistical uncertainty even at $3000 / \mathrm{fb}$ [10].

\section{Projection on Double Higgs Boson Searches}

The measurement of the Higgs boson self coupling is a fundamental test of the SM. In this context, a extremely small cross section is predicted for the main double Higgs bosons $(\mathrm{HH})$ production mechanism, (gluon fusion) $\sigma_{S M}=33 \mathrm{fb}$ at NNLO of the perturbative QCD expansion [8]. Because of such small cross section, arising from the interference of the two loop induced processes involved at the tree level (associated double Higgs production and self coupling), LHC experiments are not yet sensitive to SM HH production with the current data. The best present upper limit based on in Run 2 dataset is given by ATLAS that excluded at 95\% confidence level a signal strength $\mu \geq 7$ [12]. However, BSM effects can strongly modify the $\mathrm{HH}$ production cross section and kinematics and be observed at the LHC and, at higher scale, at HL-LHC.

The direct production of new resonant states (called $\mathrm{X}$ in the following) decaying to $\mathrm{HH}$, or resonant production, is predicted in many models such as scalars sectors extended with a doublet or a singlet, or extra dimension theories. Although different in motivation, they result in a similar experimental signature, but require experimentally the study of a broad $m_{X}$ range to be sensitive to a large variety of models. This results effectively in anomalous Higgs boson couplings that affect the non-resonant production, and may manifest with large modifications of both the cross section and the kinematic properties of the double Higgs boson final state. The exploration of many HH decay channels is crucial in this context to ensure a broad coverage of the possible BSM effects thanks to the complementarity of the different final states.

The extrapolations from Run II to HL-LHC luminosity are based on 2.3-2.7/fb of 2015 CMS dataset and shown in Fig. 3 [10], with the projections for the four final states currently under scrutiny by the CMS collaboration: $H H \rightarrow \gamma \gamma b b, H H \rightarrow \tau \tau b b, H H \rightarrow 4 b$ as well as $H H \rightarrow V V b b$, the latter looking at the $l l v v$ final state with $l=e, \mu$ and where $b$ is a jet induced by a b-quak ${ }^{1}$. The most sensitive channel comes from the decay mode of $H H \rightarrow \gamma \gamma b b$ with

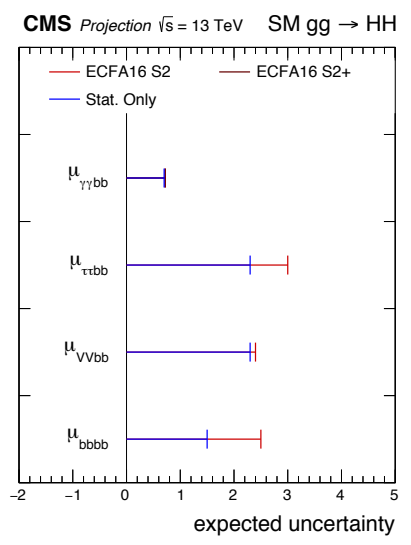

Figure 3. Projection of the sensitivity to the $\mathrm{SM} g g \rightarrow H H$ production at $3000 / \mathrm{fb}$, based on $13 \mathrm{TeV}$ preliminary analyses performed with data collected in 2015 . The uncertainty in the signal modifier $\mu=\sigma / \sigma_{S M}$ is provided assuming different scenarios on the systematic uncertainties.

a significance of 1.47 standard deviations when only considering statistical uncertainty. The

\footnotetext{
${ }^{1}$ The extrapolations presented in this document assume $\sqrt{s}=13 \mathrm{TeV}$. However, the nominal center-of-mass energy at the HL-LHC is $\sqrt{s}=14 \mathrm{TeV}$ with a predicted cross section of $39.51 \mathrm{fb}$, corresponding to an increase in cross section by $18 \%$. Assuming a background scaling with the center of mass energies (14/13 1.08), the projected results are expected to underperform by $1.18 / \sqrt{(1.08)} \sim 15 \%$ with respect to the HL-LHC energy conditions.
} 
scenario assumes uncertainty on the reconstructed b-tagged jet of $1 \%$ level and upgraded detector performance with an average of 200 pileup events. As shown, each of the channel alone is insufficient to observe the Higgs boson pair production. However, combining with these channels including other decay modes is promising.

In the $H H \rightarrow \tau \tau b b$ channel two complementary approaches have been pursued by extrapolating the projection using the full 2016 CMS dataset (36/fb) and running the full analysis on the signal and background Monte Carlo samples that incorporates the HL-LHC and Phase 2 CMS detector upgrades and operation conditions. Both the procedures are found to obtain compatible results, namely a cross section ratio $\sigma_{H H} / \sigma_{S M} \sim 1.6$ [3]. The distribution of the final discriminant used to extract the the results, the stransverse mass that is defined in [9], is showed in Fig.4 normalized to the full Phase 2 expected luminosity. Similar studies are ongoing in the other channels.

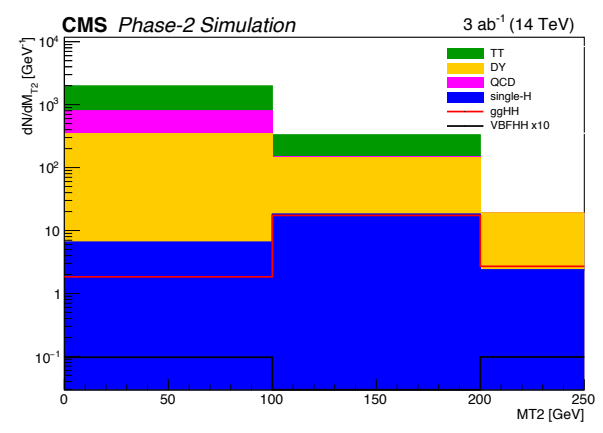

Figure 4. Distribution of the stransverse mass, defined as the largest mass of the parent particle that is compatible with the kinematic constraints of the

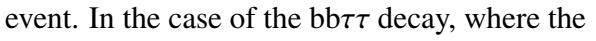
dominant background is $t \bar{t}$ production, the parent particle is interpreted as the top quark that decays into a bottom quark and a $\mathrm{W}$ boson.

\section{Rare process}

The measurement of the Yukawa couplings of the Higgs boson to fermions is one of the important studies to check the consistency with the SM and to search for possible deviations. The $H \rightarrow \mu \mu$ process can serve as a probe for coupling to fermion of second generation, provided that sufficient luminosity is collected to account for the small branching fraction $\left(2.2 \times 10^{-4}\right.$ in the SM) and reach a sizeable sensitivity. With the large datasets of the HL-LHC, searching for rare decays of the Higgs boson becomes accessible. Moreover this channel has been exploited as a benchmark for the CMS tracker upgrade. Thanks to the reduced material budget and improved spatial resolution of the upgraded tracker, about $65 \%$ better invariant mass resolution can be achieved. This leads to an uncertainty of 5\% for couplings to muons and $10 \%$ for the cross section measurement with 3000/fb [2]. Figure 5 shows the dimuon invariant mass distribution of $H \rightarrow \mu \mu$ decays for muons in the central region of the CMS detector as taken from simulated events that incorporates the CMS Phase II upgrade conditions.

\section{Search for Supersymmetric particles}

The present LHC has excluded large parts of the natural SUSY parameter space; limits for strong SUSY production are above $1 \mathrm{TeV}$ and top and bottom squarks are already highly constrained. There are still many opportunities in the electroweak sector though which may be the dominating sector if squarks and gluinos are heavy. In most SUSY breaking scenarios the supersymmetric partners of the electroweak gauge bosons (EWK-inos) are expected to have a mass of the order $100 \mathrm{GeV}$ based on naturalness. Given that EWK-inos are produced via 


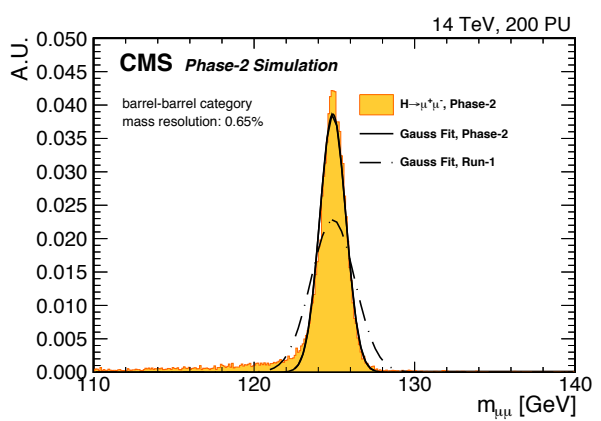

Figure 5. The di-muon invariant mass distribution for $H \rightarrow \mu \mu$ decays for muons in the central region, simulated with the Phase-2 detector.

EWK production the cross sections are small and HL-LHC has a large potential to increase sensitivity.

A brand-new CMS study for EWK-inos exploits the striking signature of same-sign leptons. It searches for mass degenerate $\tilde{\chi}_{4}^{0}, \tilde{\chi}_{2}^{ \pm}$are then expected to decay into Higgsinos (which are taken to be the lightest SUSY particles) emitting same charge W bosons with a total branching ratio close to $25 \%$ (Fig. 6).

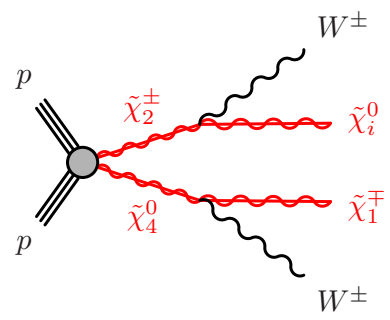

Figure 6. Example signal process which yields two same-sign leptons and large missing energy in the final state.

Given the small mass difference, the $\mathrm{W}$ bosons are soft, thus decaying into leptons with very low $p_{T}$ that are quite challanging to detect both at the trigger and offline reconstruction level. The full analysis has been performed using the fast simulation tool Delphes [13], that accounts for the performance of the upgraded CMS detector on the reconstructed final state objects. Main benefits come from the upgraded high-granularity calorimeters that improved the missing energy measurement and the extended Muon system acceptance, that yields factor 3 reduction of the irreducible WZ background. Two Higgisno mass scenarios considered:

- $\mathrm{m}\left(\tilde{\chi}^{1}\right)=150 \mathrm{GeV}$ is representative of the region of the parameter space outside the reach of the Run 2

- $\mathrm{m}\left(\tilde{\chi}^{1}\right)=250 \mathrm{GeV}$ is outside the sensitivity reach of the same search when extrapolated to the HL-LHC

The spectrum of the transverse mass of the lepton candidate (built by combining the lepton $p_{T}$ and missing energy), is shown in Fig.7 top panel. Wino-like mass degenerate $\tilde{\chi}_{4}^{0}, \tilde{\chi}_{2}^{ \pm}$are excluded at $95 \% \mathrm{CL}$ up to $900 \mathrm{GeV}$ in the tested mass scenarios as shown in Fig. 7 (bottom panel).

\section{Exotic Searches}

It may happen that the only hints of new physics are rather exotic signatures that cannot be detected with conventional analyses New physics could manifest itself with a non-standard 

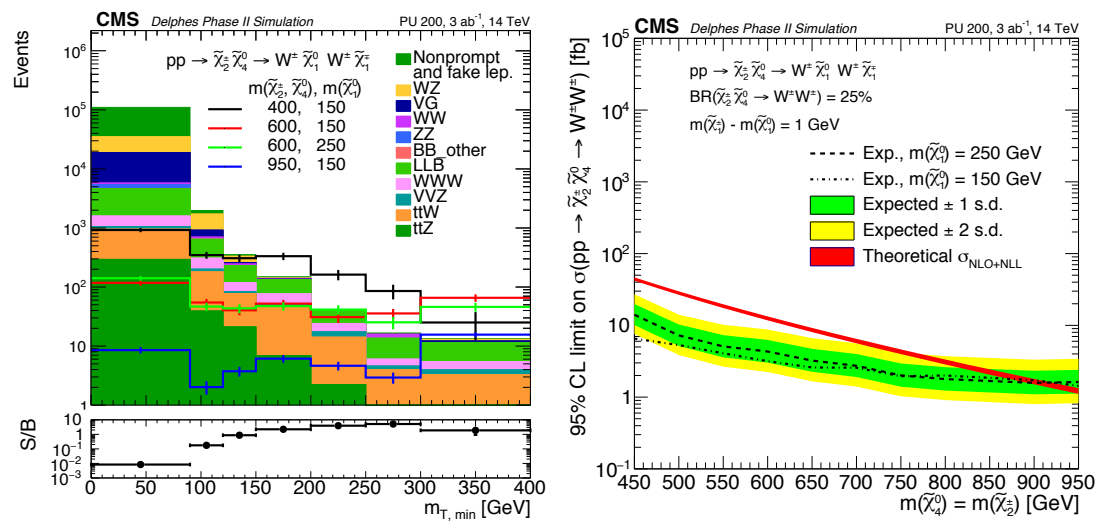

Figure 7. Top panel: the discriminating variable $\mathrm{mT}$,min reconstructed from the leptons and missing transverse energy. The seven bins would allow an extraction of the signal from the background. Botton panel: exclusion limit in case of no observation of a signal.

signature, requiring dedicated trigger and reconstruction algorithms and specific detector features. One example are slow moving particles, also known as Heavy Stable Charged Particles (HSCP) foreseen by Split SUSY scenarios. These particles feature very high mass and small velocity. One way to detect such particle is their anomalous energy loss $(\mathrm{dE} / \mathrm{dx})$ [11] provided they are triggered. Muons from the decay of slow moving particles can already be identified at the trigger level based on the difference in arrival time with respect to relativistic SM particles. The muons from such decays are delayed w.r.t. to the time-zero bunch crossing (BX) and will spill over in neighbouring BXs. Fig. 8 (left) illustrates such a time difference as seen by CMS RPC chambers instrumenting the barrel and the forward muon system. The ns time resolution of the upgraded muon trigger allows to detect slowly moving charged particles by their hits. This will significantly extend the trigger capability to very slow particles with beta $\leq 0.5$ (red) compared to present conditions, as displayed in Fig. 8 right [4].
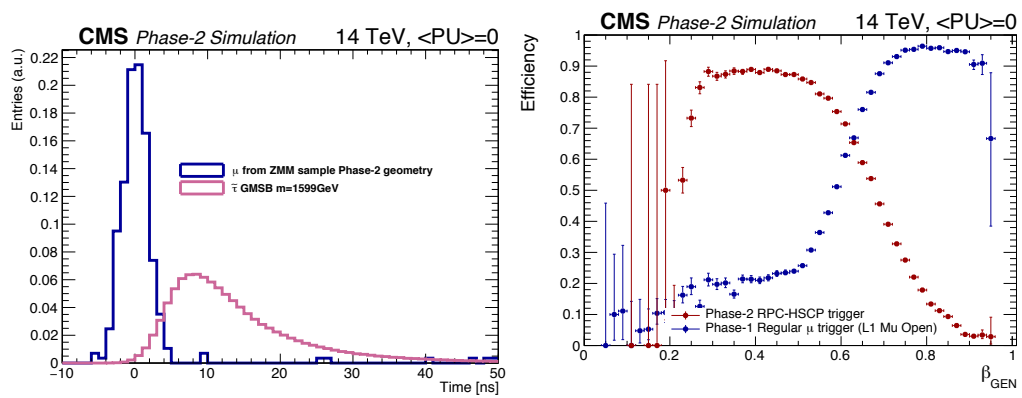

Figure 8. Left: measured time for a particle to traverse CMS with respect to the transit time of a particle traveling at the speed of light. Muons from the decay of $\mathrm{Z}$ bosons are relativistic $(\beta=1)$ and HSCPs are slower. Right: the efficiency as a function of $b$ of the standard L1 muon trigger without any $\mathrm{pT}$ threshold, and the RPC-HSCP Phase-2 trigger with 1.56 ns sampling time

An important class of Gauge Mediated SUSY breaking models predict long-lived particles that would potentially lead to displaced signatures. For particles of a few hundred GeV 
mass, impact parameters can reach up to approximately one meter (or longer) for sufficiently large lifetimes In this context, an interesting benchmark channel studied in the context of the CMS Phase II upgrade is the search for the supersymmetric partners of the muons (s-muons) that can be the co-NLSP (i.e. they are the next to lightest LSP) and almost degenerate in mass. This search has low sensitivity in the present LHC runs, given the extremely small cross section, around $10^{-2} \mathrm{fb}$ for $1 \mathrm{TeV}$ s-muon masses but became accessible in the HL-LHC scenario. The search sensitivity depends on the s-muons decay lengths: larger displacement (driven by

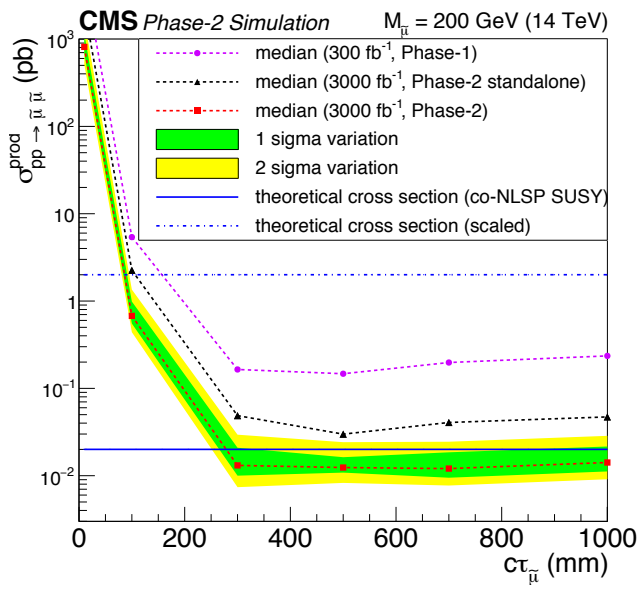

Figure 9. The 95\% CL upper limits of $q q \rightarrow \tilde{\mu} \tilde{\mu}$ with $\tilde{\mu}$ to a muon and a gravitino as a function of the decay length for s-muon mass of $200 \mathrm{GeV}$. The theoretical cross section for the specific model is represented by the blue solid line. For different SUSY breaking scales, $\tan \beta$ or otherwise modified parameters, the cross sections may be 100 times larger, reflected by the blue dash-dotted line. Green (yellow) shaded bands show the one (two) sigma range of variation of the expected 95\% CL limits. Phase II results with an average 200 pileup events and an integrated luminosity of $3000 / \mathrm{fb}$ are compared to results obtained with $300 / \mathrm{fb}$. The black line shows the sensitivity without the muon reconstruction algorithm dedicated to the displaced muon and mainly relying on the muon system chambers. Using the standard muon reconstruction algorithms reduces the reconstruction efficiency by a factor three.

longer s-muon lifetimes) allow to test a phase space region that is free from the SM background. In the proposed benchmark channel, the s-muon decay to a SM muon and a gravitino (whose signature in the detector is the missing energy) with lifetimes which can be very long such that the displacement is of the order of meter. Muon triggering and reconstruction can be performed by the muon system in stand-alone, without the tracker. Discovery sensitivity of $3 \sigma$ significance can be reached with $3000 \mathrm{~b}$ of data (Fig. 9), providing full trigger and offline reconstruction efficiency even in the pile-up 200 environment.

\section{Conclusions}

In the middle of 2026, LHC will reach a peak instantaneous luminosity of $7.5 \times 10^{34} \mathrm{~cm}-2 \mathrm{~s}-1$. The HL-LHC will be the first Higgs factory, opening up the possibility to perform high precision measurement thanks to the to large amounts of events that is planned to collect, corresponding to an integrated luminosity of $3000 / \mathrm{fb}$, more than 10 times larger than data by the end of Run 3. Thanks to the high statistics, rare processes in the Higgs sector will become accessible as well as the possibility to study the any possible deviation from the Standard Model. Towards the high precision era, a key role will be thus played by the theoretical and experimental systematics. The large Phase 2 dataset will enrich the potential to test physics beyond the SM, providing that the CMS experiment will be able to cope the challenging operation conditions. An extensive detector upgrade program has been planned and will start around 2023. Long-term exploration of Higgs sector and New Physics searches at HL-LHC will have crucial impact on our understanding of nature. There is a coordinated effort by ATLAS, CMS and theorists to update and combine results which will be documented in a 
CERN Yellow Report at the end of the year. Better precisions should come from the optimized Phase- 2 detectors documented in TDRs and from the better analysis tools developed for Run 2.

\section{References}

[1] CMS Collaboration, CERN-LHCC-2015-10, https://cds.cern.ch/record/2020886

[2] CMS Collaboration, CMS-TDR-17-001, https://cds.cern.ch/record/2272264.

[3] CMS Collaboration, CMS-TDR-019, https://cds.cern.ch/record/2293646

[4] CMS Collaboration, CMS-TDR-17-003

[5] CMS Collaboration, CMS-PAS-HIG-17-031, 2018, http://cds.cern.ch/record/2308127

[6] F. Bishara et al., arXiv:1606.09253v3

[7] CMS Collaboration, CMS-HIG-17-028, https://cds.cern.ch/record/2628757

[8] D. de Florian et. al., 10.23731/CYRM-2017-002, arXiv:1610.07922

[9] A. J. Barr, M. J. Dolan, C. Englert, and M. Spannowsky, Phys. Lett. B 728 (2014) 308.

[10] CMS Collaboration, CMS-PAS-FTR-16-002, http://cds.cern.ch/record/2266165.

[11] CMS Collaboration, CMS-PAS-EXO-14-007

[12] ATLAS Collaboration, ATLAS-CONF-2018-043

[13] The DELPHES 3 Collaboration, de Favereau, J., Delaere, C. et al. J. High Energ. Phys. (2014) 2014: 57, https://doi.org/10.1007/JHEP02(2014)057 